\title{
Screening of Sunflower (Helianthus annuus L) Genotypes for Moisture Stress Tolerance using PEG-6000
}

\author{
Sheshaiah $^{1}$ *, I. Shankergoud ${ }^{2}$, R. Siddhesh, Nadkarni ${ }^{3}$ and Vikas V. Kulakarni ${ }^{4}$ \\ ${ }^{1}$ Department of Genetics and Plant Breeding, CoA, UAHS, Shivamogga-577225, India \\ ${ }^{2}$ Directorate of Research, University of Agricultural Sciences, Raichur-584 104, India \\ ${ }^{3}$ Department of Genetics and Plant Breeding, College of agriculture Vellayani, Kerala \\ Agricultural University, Trivedrum-695522, India \\ ${ }^{4}$ Scientist (Breeder), AICRP on Sunflower, MARS, UAS, Raichur-584 104, India \\ *Corresponding author
}

\section{A B S T R A C T}

An in-vitro experiment was conducted using Polyethylene glycol-6000 (PEG-6000) at MARS, UAS, Raichur, to identify genotypes which overcome moisture stress condition at germination and seedling establishment stage of Sunflower based on stress tolerance

\begin{tabular}{|l|}
\hline Ke y w or d s \\
PEG-6000, \\
GTI, \\
RLTI, \\
PHTI and \\
SLTI \\
\hline Article Info \\
\hline Accepted: \\
04 April 2017 \\
Available Online: \\
10 May 2017 \\
\hline \hline
\end{tabular}
index. A total of 160 sunflower genotypes in two replications were screened in petri-dishes at six water stress levels $(0,10,15,20,25$ and $30 \%$ of PEG-6000) induced by PEG-6000 in two factorial CRD fashion. The germination percentage, shoot length, root length and seedling length were recorded on seven days after of sowing, later these observations are converted into the germination stress tolerance index (GTI), root length stress tolerance index (RLTI), plant height stress tolerance index (PHTI) and seedling length stress tolerance index (SLTI). The results of analysis of variance showed that, all treatments, genotypes and G X T interactions were significant for all the studied traits. The treatment means indicated that GTI, RLTI, PHTI and SLTI are drastically reduced with the increased percentage of PEG. The genotype $\mathrm{x}$ treatment interactions at 20 and 25\% PEG few genotypes exhibited significant differences for studied traits, hence, these concentrations are considered as critical doses to isolate the real moisture tolerant genotypes. Based on per se mean performance for GTI, RLTI, PHTI and SLTI, there are 34 promising genotypes were identified as a moisture stress tolerant genotypes.

\section{Introduction}

The sunflower (Helianthus annuus) is an annual oilseed crop in the family Asteraceae and it is valued and healthy vegetable oil. Sunflower plant growth and productivity is limited by several biotic and abiotic stresses. Among the abiotic stresses, moisture stress or drought is most important stress, which affecting germination, seedling establishment, flowering and seed filling stages of sunflower. Seed germination is the most sensitive stage to moisture stress in the plant life cycle (Ashraf and Mehmood, 1990) and it is foremost stage to maintain adequate population of the crop for obtaining prominent yield, in order to overcome such situation. Hence, an experiment undertook to identify genotypes which are suitable to grow under moisture stress conditions through germination test under laboratory condition using PEG-6000. 
PEG-6000 is moisture stress inducing agent having a molecular weight of 6000 . Using PEG-6000 Ahmad et al., (2009) imposed drought stress at germination and seedling growth stages at five water stress levels and identified the two best stress tolerant sunflower genotypes viz., G-101 and 64-A-93 from in vitro response of PEG-6000. PEG based screening of genotypes for moisture stress tolerance is a complementary method to field screening (Ahmad, 2009 and Geetha et al., 2012) because it overcome the difficulties of filed trails like uncontrolled climatic conditions, heterogeneity of soil, large number of germplasm and labor consumption. Turhan and Baser (2004) also opined that in vitro approach could be useful in screening and selecting for drought response prior to field trial. Hence, in the present investigation PEG-6000 used to develop moisture stress under in vitro condition mainly to study the response of genotypes to moisture stress condition.

\section{Materials and Methods}

In vitro screening of genotypes is an alternate and simple method to screen large number of genotypes by way of limited space and in this experiment, a total 160 genotypes (12 maintainers, 12 restorers and 136 germplasm lines) collected from Indian Institute of Oilseed Research, Hyderabad were tested for moisture stress response at MARS, UAS, Raichur, Karnataka. PEG-6000 is a water stress stimulator and there are six moisture stress treatments of 0 (control), -0.6 (10\%), $0.9(15 \%),-1.2(20 \%),-1.5(25 \%)$ and -1.8 (30\%) M Pa was imposed by dissolving 0 , $10,15,20,25$ and $30 \mathrm{~g}$ of PEG-6000 in 100 $\mathrm{ml}$ of distilled water. Two replications were maintained for each osmotic potential and the experiment was laid out in two factorial-CRD fashion. Twenty seeds of each genotype were rinsed and soaked separately for ten minutes using distilled water. The seeds were kept on the germinating paper in each petri plate, and then treated ten $\mathrm{ml}$ of designated treatment solution was applied daily in each Petri plate after washing out the previous solution, separately.

The germination percentage, root length, shoot length and seedling length were recorded on five randomly selected plants seven days after sowing and from these measurements, germination stress tolerance index, root length stress tolerance index, plant height stress tolerance index and seedling length stress tolerance index were calculated using the following formulae given by Ashraf et al., (2006).

Germination

tolerance index $(\%)$

stress Number of seeds germinated

$=$ Total number of seeds kept for germination x 100

Root length stress tolerance index $=\frac{\text { Root length stressed plants }}{\text { Root length of control plants }} \times 100$

Plant height stress tolerance index $=\frac{\text { Plant height of stressed plants }}{\text { Plant height of control plants }} \times 100$

Seedling length stress tolerance index $=\frac{\text { Seedling length of stressed plants }}{\text { Seedling length of control plants }} \times 100$ 


\section{Results and Discussion}

The analysis of variance for the data on 160 sunflower genotypes under laboratory screening using PEG-6000 is presented in the Table 1 . The mean sum of squares of analysis of variance exhibited that, all the treatments, genotypes and genotypes $\mathrm{x}$ treatment interactions are found to be highly significant for all the studied characters under laboratory condition, this indicates that sunflower germplasm differed significantly for their responses in used in the study. Highly Significant differences were reported by Ahmad et al., (2009) for GSI, PHSI and RLSI in the ANOVA.

The obtained treatment mean effects for characters are presented in Table 2. The significant differences were noticed for most of the studied characters at 10 and 15 per cent concentration of PEG-6000. At 20 per cent of PEG-6000 concentration, significant differences observed for GSI and RLSI. At 25 and at 30 per cent PEG-6000 concentration, none of the character found significant. The overall treatment means effects indicated that GSI, RLSI, PHSI and SLSI drastically reduced with increased concentrations of PEG-6000. These results are accordance with Ahmad et al., (2009) and Saensee (2011) for GSI, PHSI and RLSI.

The results of the genotype $\mathrm{x}$ treatment interaction of 160 sunflower genotypes under laboratory evaluation are presented in Table 3. The per se performance of interaction effect of 160 sunflower genotypes are screened at $0,10,15,20,25$ and 30 per cent concentration of PEG-6000. The results revealed that at 10 and 15 per cent concentrations (data not shown) majority of the genotypes showed significant mean per se performance. At 30 per cent PEG-6000 concentration (data not shown) majority of the genotypes could not survive and could not exhibit significant per se performance for many of the characters and this concentration acted as lethal doses. However, few genotypes could express significant difference for studied traits at 20 and 25 per cent of PEG-6000, and are presented in Table 4.

The effect of water stress created by PEG6000 indicated that germination stress tolerance index is varied among 160 genotypes and it is ranged from 0 to 100 . At 20 per cent concentration 106 out of 160 genotypes showed significant per se performance, while, at 25 per cent concentration 28 genotypes were found to be significant and highest GSI were recorded for $\mathrm{GP}_{6}-2255$ and $\mathrm{GP}_{6}-305$ (95.00 \%). Among CMS lines, CMS 857B, among restorer lines, R-12-2, R-78 and among germplasm lines $\mathrm{GP}_{6^{-}}-11, \mathrm{GP}_{6}-54, \mathrm{GP}_{6}-118, \mathrm{GP}_{6}-211, \mathrm{GP}_{6}-305$, $\mathrm{GP}_{6}-310, \mathrm{GP}_{6}-326, \mathrm{GP}_{6}-366, \mathrm{GP}_{6}-370, \mathrm{GP}_{6}-$ 371, $\mathrm{GP}_{6}-424, \mathrm{GP}_{6}-442, \mathrm{GP}_{6}-578, \mathrm{GP}_{6}-614$, $\mathrm{GP}_{6^{-}}$-714, $\mathrm{GP}_{6}-863, \mathrm{GP}_{6^{-}}-912, \mathrm{GP}_{6}-967, \mathrm{GP}_{6^{-}}$ 969, $\mathrm{GP}_{6}-1060, \mathrm{GP}_{6}-1072, \mathrm{GP}_{6}-1102, \mathrm{GP}_{6^{-}}$ 1228, $\mathrm{GP}_{6}-1576$ and $\mathrm{GP}_{6}-2255$ exhibited significant per se performance.

The RLSI under all PEG concentrations worked as per the formula given in the materials and methods and it is ranged from 0 to 744.41. At 20 per cent concentration indicated 43 out of 160 genotypes were showed significant per se performance, while, at 25 per cent concentration six genotypes found to be significant and the highest was recorded for CMS 857B (417.58 \%). Among CMS lines, CMS 104B and CMS 857B, among germplasm lines, GP6-325, GP6-586, GP6-714 and GP6-589 exhibited significant per se performance. And among restorer lines, none of the lines exhibited significant effects. The increased root length stress index with increased level of PEG-6000 was observed upto15 \% concentrations but at higher concentration (20-30\%) observed the decreased performance, these results 
accordance with reports of Ahmad et al., (2009) and Saense et al., (2012).

The PHSI is also varied among 160 sunflower genotypes studied and it was ranged from 0 to 93.72. The plant height stress tolerance index at 20 per cent concentration indicated 11 out of 160 genotypes showed significant per se performance, while at 25 per cent concentration none of the genotypes are found to be significant because the shoot length most affected apart from germination and root length. The PHTI decreased with increased PEG-6000 concentrations, these results accordance with reports of Ahmad et al., (2009) and Saense et al., (2012).
The Seedling length stress tolerance index (SLTI) at 20 and 25 per cent concentration of PEG-6000 was also worked out and it is ranged from $0.00-282.85$. At 20 per cent concentration indicated 43 out of 160 genotypes were showed significant per se performance, while, at 25 per cent concentration six genotypes found to be significant and top performing genotype is CMS 857B (137.45\%). Among CMS lines, CMS 104B and CMS 857B, among germplasm lines, GP6-325, GP6-586, GP6589 and GP6-714 exhibited significant per se performance. And among restorer lines, none of the lines exhibited significant effects.

Table.1 Analysis of variance for eight different characters in sunflower under laboratory evaluation using PEG-6000

\begin{tabular}{|c|c|c|c|c|c|}
\hline Source & DF & GTI & RLTI & PHTI & SLTI \\
\hline Treatment & 4 & $422295.32^{* *}$ & $1220734.18^{* *}$ & $133502.02^{* *}$ & $476047.95^{* *}$ \\
\hline Genotypes & 159 & $1765.31^{* * *}$ & $22119.52^{* *}$ & $564.30^{* *}$ & $3923.39^{* *}$ \\
\hline Treatment x Genotype & 636 & $382.65^{* *}$ & $2830.30^{* *}$ & $129.076^{* *}$ & $573.75^{* *}$ \\
\hline Error & 800 & 18.92 & 101.60 & 10.60 & 14.88 \\
\hline
\end{tabular}

* - significant at $5 \%$ probability; ** - significant at $1 \%$ probability

Table.2 Treatment effect means of different concentrations of PEG-6000 for characters in sunflower

\begin{tabular}{|c|c|c|c|c|}
\hline Treatment & GTI & RLTI & PHTI & SLTI \\
\hline $10 \%$ & $95.15^{* *}$ & $152.02^{* *}$ & $52.48^{* * *}$ & $97.33^{* * *}$ \\
\hline $15 \%$ & $89.52^{* *}$ & $133.06^{* *}$ & $30.24^{* *}$ & $76.35^{* *}$ \\
\hline $20 \%$ & $74.49^{* *}$ & $84.54^{*}$ & 14.99 & 46.46 \\
\hline $25 \%$ & 41.49 & 37.54 & 7.01 & 20.29 \\
\hline $30 \%$ & 8.65 & 6.05 & 1.99 & 3.88 \\
\hline Mean & 61.86 & 82.64 & 21.34 & 48.86 \\
\hline S.Em+/- & 0.24 & 0.56 & 0.18 & 0.21 \\
\hline CD 5\% & 0.67 & 1.56 & 0.50 & 0.59 \\
\hline CD 1\% & 0.88 & 2.05 & 0.66 & 0.78 \\
\hline CV & 7.03 & 12.19 & 15.25 & 7.89 \\
\hline
\end{tabular}

* - significant at 5\% probability

** - significant at $1 \%$ probability 
Table.3 Genotype x treatment interaction effects of GTI, RLTI, PHTI and SLTI at 20 and $25 \%$ of PEG-6000

\begin{tabular}{|c|c|c|c|c|c|c|c|c|c|}
\hline \multirow{3}{*}{$\begin{array}{c}\text { Sl. } \\
\text { No. }\end{array}$} & \multirow{3}{*}{ Genotypes } & \multirow{2}{*}{\multicolumn{2}{|c|}{$\begin{array}{c}\text { GTI } \\
\text { PEG 6000 } \\
\text { concentrations }\end{array}$}} & \multirow{2}{*}{\multicolumn{2}{|c|}{$\begin{array}{c}\text { RLTI } \\
\text { PEG 6000 } \\
\text { concentrations }\end{array}$}} & \multirow{2}{*}{\multicolumn{2}{|c|}{$\begin{array}{c}\text { PHTI } \\
\text { PEG 6000 } \\
\text { concentrations }\end{array}$}} & \multirow{2}{*}{\multicolumn{2}{|c|}{$\begin{array}{c}\text { SLTI } \\
\text { PEG 6000 } \\
\text { concentrations }\end{array}$}} \\
\hline & & & & & & & & & \\
\hline & & $20 \%$ & $25 \%$ & $20 \%$ & $25 \%$ & $20 \%$ & $25 \%$ & $20 \%$ & $25 \%$ \\
\hline 1 & CMS-17B & 48.57 & 0.00 & 56.42 & 0.00 & 14.40 & 0.00 & 35.37 & 0.00 \\
\hline 2 & CMS-103B & 55.56 & 38.89 & 76.41 & 55.64 & 25.57 & 18.53 & 50.84 & 37.07 \\
\hline 3 & CMS-104B & $74.34^{* * *}$ & 33.29 & $296.54^{* * *}$ & $140.60^{* * *}$ & 25.94 & 13.00 & $137.90^{* * *}$ & $65.82^{* * *}$ \\
\hline 4 & CMS-148B & $100.00^{* * *}$ & 70.00 & $177.06^{\text {*** }}$ & 59.41 & 16.99 & 9.44 & $66.64^{* * *}$ & 25.12 \\
\hline 5 & CMS-335B & $90.00^{* * *}$ & 65.00 & 60.68 & 44.07 & 16.86 & 9.53 & 38.04 & 26.25 \\
\hline 6 & CMS-338B & $90.00^{* * *}$ & 45.00 & 57.00 & 20.30 & 26.53 & 9.11 & 40.57 & 14.29 \\
\hline 7 & CMS-351B & $85.00^{* * *}$ & 40.00 & 72.51 & 2.16 & 20.58 & 6.67 & 53.78 & 3.79 \\
\hline 8 & CMS-378B & $75.00^{* * *}$ & 45.00 & 97.46 & 36.54 & 9.15 & 3.63 & 43.46 & 16.46 \\
\hline 9 & CMS-607B & $70.47^{*}$ & 0.00 & 28.08 & 0.00 & 14.97 & 0.00 & 21.54 & 0.00 \\
\hline 10 & CMS-850B & $95.00^{* * *}$ & 45.00 & 54.51 & 17.90 & 16.80 & 5.63 & 33.40 & 11.09 \\
\hline 11 & CMS-852B & $95.00^{* * *}$ & 65.00 & 49.44 & 14.29 & 14.48 & 6.19 & 31.83 & 10.20 \\
\hline 12 & CMS-857B & $95.00^{* * *}$ & $90.00^{* * *}$ & $522.92^{* * *}$ & $417.58^{* * *}$ & 19.97 & 16.11 & $172.01^{* * *}$ & $137.45^{*}$ \\
\hline 13 & R-12-2 & $100.00^{\text {*** }}$ & $80.00^{\text {*** }}$ & $128.01^{* * *}$ & 55.78 & $37.78^{* * *}$ & 14.12 & $86.13^{* *}$ & 36.42 \\
\hline 14 & R 127-1 & $94.74^{* * *}$ & 29.82 & $159.64^{\text {*** }}$ & 68.31 & 16.80 & 3.70 & 40.40 & 14.44 \\
\hline 15 & R 64NB & $82.50^{* * *}$ & 60.00 & 96.04 & 40.15 & 23.14 & 11.15 & $59.20^{* * *}$ & 25.58 \\
\hline 16 & R-78 & $95.00^{* * *}$ & $87.50^{* * *}$ & 95.74 & 80.02 & 21.68 & 16.94 & $61.37^{* *}$ & 50.72 \\
\hline 17 & R-630 & $87.50^{* * *}$ & 37.50 & 90.74 & 53.89 & 20.99 & 10.75 & $58.35^{*}$ & 33.87 \\
\hline 18 & GM-27R & $77.50^{* * *}$ & 48.33 & 36.24 & 25.37 & 23.55 & 16.62 & 30.16 & 21.19 \\
\hline 19 & GM-37R & $81.29^{* *}$ & 8.19 & 46.17 & 26.42 & $27.77^{*}$ & 13.39 & 37.61 & 20.37 \\
\hline 20 & GM-41R & $95.00^{* * *}$ & 50.00 & 81.49 & 58.82 & 23.92 & 11.25 & 50.26 & 33.01 \\
\hline 21 & GM-44R & 69.85 & 66.54 & 77.73 & 63.67 & 25.80 & 18.16 & 49.68 & 39.09 \\
\hline 22 & GM-56R & 61.11 & 27.78 & 64.88 & 4.01 & $40.70^{* * *}$ & 10.64 & $56.86^{*}$ & 6.21 \\
\hline 23 & GM-59R & 55.56 & 33.33 & 46.36 & 34.57 & $37.20^{* * *}$ & 14.32 & 42.53 & 26.07 \\
\hline 24 & GM-71R & $77.78^{* * *}$ & 66.67 & 51.17 & 37.40 & 16.50 & 4.50 & 36.97 & 23.93 \\
\hline 25 & $\mathrm{GP}_{6}-11$ & $90.00^{* *}$ & $90.00^{* * *}$ & 53.28 & 35.20 & 15.27 & 11.19 & 34.19 & 23.14 \\
\hline 26 & $\mathrm{GP}_{6}-18$ & $85.00^{* * *}$ & 15.00 & 53.00 & 29.76 & 11.95 & 6.43 & 32.59 & 18.19 \\
\hline 27 & $\mathrm{GP}_{6}-18-1$ & $94.74^{* * *}$ & 21.05 & 24.84 & 11.52 & 6.32 & 4.85 & 14.80 & 7.91 \\
\hline 28 & $\mathrm{GP}_{6}-54$ & $87.50^{* * *}$ & $75.00^{* * *}$ & 91.46 & 34.73 & 14.70 & 10.46 & $59.22^{* * *}$ & 24.54 \\
\hline 29 & $\mathrm{GP}_{6}-63$ & 63.16 & 23.68 & 64.07 & 19.35 & 7.83 & 4.83 & 34.09 & 11.60 \\
\hline 30 & $\mathrm{GP}_{6}-83$ & $81.58^{* * *}$ & 28.95 & $103.79^{*}$ & 51.71 & 12.69 & 12.07 & 52.12 & 29.22 \\
\hline 31 & $\mathrm{GP}_{6}-109$ & 60.00 & 10.00 & 36.00 & 7.24 & 6.85 & 3.72 & 19.64 & 5.24 \\
\hline 32 & $\mathrm{GP}_{6}-118$ & $90.00^{* *}$ & $85.00^{* * *}$ & 53.54 & 38.18 & 13.64 & 4.46 & 37.03 & 24.22 \\
\hline 33 & $\mathrm{GP}_{6}-127$ & $76.32^{* *}$ & 31.58 & 73.12 & 15.35 & $27.72^{*}$ & 7.22 & 48.52 & 10.94 \\
\hline 34 & $\mathrm{GP}_{6}-135$ & 57.89 & 15.79 & 56.16 & 15.69 & 14.91 & 5.66 & 37.24 & 11.09 \\
\hline 35 & $\mathrm{GP}_{6}-139$ & 47.22 & 25.00 & 76.04 & 21.71 & 4.80 & 1.80 & 36.65 & 10.74 \\
\hline 36 & $\mathrm{GP}_{6}-160$ & 42.11 & 0.00 & 43.18 & 0.00 & 14.85 & 0.00 & 25.00 & 0.00 \\
\hline 37 & $\mathrm{GP}_{6}-173$ & $82.50^{* * *}$ & 65.00 & 71.94 & 26.57 & 16.22 & 8.18 & 47.03 & 18.34 \\
\hline 38 & $\mathrm{GP}_{6}-175$ & 67.50 & 22.50 & 40.79 & 50.88 & 10.92 & 8.05 & 25.42 & 28.80 \\
\hline
\end{tabular}




\begin{tabular}{|c|c|c|c|c|c|c|c|c|c|}
\hline 39 & $\mathrm{GP}_{6}-176$ & 69.85 & 57.54 & 96.34 & 80.08 & 13.95 & 7.50 & 45.09 & 34.94 \\
\hline 40 & $\mathrm{GP}_{6}-181$ & 50.00 & 0.00 & $103.57^{*}$ & 0.00 & 5.15 & 0.00 & 53.81 & 0.00 \\
\hline 41 & $\mathrm{GP}_{6}-211$ & $100.00^{* * *}$ & $85.00^{* * *}$ & 94.29 & 44.48 & 13.33 & 7.48 & 55.51 & 26.76 \\
\hline 42 & $\mathrm{GP}_{6}-217$ & $90.00^{* *}$ & 7.50 & 90.44 & 11.45 & 7.43 & 6.64 & $57.16^{*}$ & 9.53 \\
\hline 43 & $\mathrm{GP}_{6}-219$ & 40.00 & 5.00 & 43.50 & 18.45 & 4.54 & 1.71 & 23.20 & 9.73 \\
\hline 44 & $\mathrm{GP}_{6}-226$ & $85.00^{* * *}$ & 32.50 & 39.41 & 23.68 & 10.02 & 4.28 & 23.36 & 13.08 \\
\hline 45 & $\mathrm{GP}_{6}-236$ & 67.50 & 55.00 & 37.33 & 12.93 & 13.75 & 9.12 & 29.14 & 11.61 \\
\hline 46 & $\mathrm{GP}_{6}-250$ & 52.63 & 15.79 & 85.12 & 39.81 & 16.84 & 8.38 & 49.14 & 23.26 \\
\hline 47 & $\mathrm{GP}_{6}-263$ & 63.16 & 7.89 & 69.04 & 13.64 & 15.78 & 6.72 & 45.89 & 10.62 \\
\hline 48 & $\mathrm{GP}_{6}-271$ & $90.00^{* * *}$ & 65.00 & $194.94^{\text {*** }}$ & 72.43 & 14.39 & 9.61 & $102.88^{\text {*** }}$ & 40.40 \\
\hline 49 & $\mathrm{GP}_{6}-276$ & $89.47^{\text {*** }}$ & 60.53 & 57.35 & 40.00 & 14.77 & 10.54 & 30.15 & 21.20 \\
\hline 50 & $\mathrm{GP}_{6}-282$ & 39.47 & 0.00 & 54.60 & 0.00 & 6.51 & 0.00 & 25.00 & 0.00 \\
\hline 51 & $\mathrm{GP}_{6}-286$ & $72.95^{*}$ & 43.27 & $156.91^{\text {** }}$ & 58.61 & 18.30 & 10.66 & $87.16^{* * *}$ & 34.49 \\
\hline 52 & $\mathrm{GP}_{6}-297$ & $82.50^{* * *}$ & 27.50 & 94.49 & 6.73 & 13.68 & 3.57 & 51.52 & 5.02 \\
\hline 53 & $\mathrm{GP}_{6}-303$ & 0.00 & 0.00 & 0.00 & 0.00 & 0.00 & 0.00 & 0.00 & 0.00 \\
\hline 54 & $\mathrm{GP}_{6}-305$ & $100.00^{* *}$ & $95.00^{* * *}$ & $141.48^{* *}$ & 60.62 & 9.77 & 3.89 & $66.24^{* * *}$ & 28.21 \\
\hline 55 & $\mathrm{GP}_{6}-310$ & $92.50^{* * *}$ & $87.50^{* * *}$ & 36.31 & 22.99 & 10.88 & 6.52 & 21.54 & 13.42 \\
\hline 56 & $\mathrm{GP}_{6}-312$ & 52.50 & 27.50 & 72.14 & 19.28 & 8.30 & 5.19 & 38.87 & 11.94 \\
\hline 57 & $\mathrm{GP}_{6}-313$ & 25.00 & 0.00 & 14.35 & 0.00 & 3.98 & 0.00 & 8.73 & 0.00 \\
\hline 58 & $\mathrm{GP}_{6}-317$ & 27.50 & 0.00 & 36.62 & 0.00 & 10.34 & 0.00 & 22.99 & 0.00 \\
\hline 59 & $\mathrm{GP}_{6}-324$ & $84.21^{* * *}$ & 50.00 & 89.73 & 18.84 & 11.47 & 5.64 & 51.12 & 12.34 \\
\hline 60 & $\mathrm{GP}_{6}-325$ & $87.50^{\text {*** }}$ & 62.50 & $195.38^{* * *}$ & $105.23^{*}$ & 9.69 & 5.29 & $113.23^{* * *}$ & $60.97^{* *}$ \\
\hline 61 & $\mathrm{GP}_{6}-326$ & $100.00^{* * *}$ & $80.00^{* * *}$ & $159.36^{* * *}$ & 70.34 & $34.00^{* * *}$ & 11.04 & $74.85^{* * *}$ & 30.40 \\
\hline 62 & $\mathrm{GP}_{6}-327$ & $77.50^{* * *}$ & 52.50 & 97.74 & 35.74 & 21.07 & 7.13 & 52.18 & 18.74 \\
\hline 63 & $\mathrm{GP}_{6}-331$ & $82.50^{* * *}$ & 57.50 & $128.91^{\text {*** }}$ & 45.13 & 23.53 & 11.44 & $81.83^{* * *}$ & 30.09 \\
\hline 64 & $\mathrm{GP}_{6}-332$ & $84.21^{* * *}$ & 50.00 & 49.00 & 20.69 & 10.33 & 5.30 & 36.50 & 15.72 \\
\hline 65 & $\mathrm{GP}_{6}-347$ & $90.00^{* * *}$ & 30.00 & 31.97 & 9.27 & 9.08 & 6.63 & 20.27 & 7.94 \\
\hline 66 & $\mathrm{GP}_{6}-358$ & 34.21 & 13.16 & 69.10 & 42.10 & 14.36 & 13.40 & 44.62 & 29.27 \\
\hline 67 & $\mathrm{GP}_{6}-366$ & $86.84^{* * *}$ & $81.58^{* * *}$ & $123.35^{* * *}$ & 80.34 & 27.67 & 16.05 & $74.62^{\text {*** }}$ & 47.59 \\
\hline 68 & $\mathrm{GP}_{6}-370$ & $100.00^{* * *}$ & $75.00^{* * *}$ & 44.47 & 15.95 & 16.81 & 9.17 & 35.64 & 13.78 \\
\hline 69 & $\mathrm{GP}_{6}-371$ & $95.00^{* * *}$ & $92.50^{* *}$ & $120.03^{\text {*** }}$ & 64.50 & 14.76 & 4.39 & $61.14^{* * *}$ & 30.87 \\
\hline 70 & $\mathrm{GP}_{6}-374$ & $90.63^{* * *}$ & 65.63 & 72.03 & 33.86 & 26.08 & 15.55 & 50.96 & 25.49 \\
\hline 71 & $\mathrm{GP}_{6}-384$ & $72.50^{*}$ & 52.50 & 43.48 & 17.39 & 8.03 & 4.31 & 29.65 & 12.29 \\
\hline 72 & $\mathrm{GP}_{6}-387$ & $90.00^{* * *}$ & 25.00 & 68.59 & 39.43 & 11.65 & 6.72 & 39.38 & 22.65 \\
\hline 73 & $\mathrm{GP}_{6}-400$ & $86.11^{* * *}$ & 47.22 & 61.23 & 31.65 & 9.74 & 7.75 & 32.45 & 18.32 \\
\hline 74 & $\mathrm{GP}_{6}-420$ & 70.00 & 55.00 & 84.09 & 42.18 & 7.55 & 3.40 & 42.32 & 21.01 \\
\hline 75 & $\mathrm{GP}_{6}-424$ & $95.00^{* * *}$ & $92.50^{* * *}$ & $187.31^{\text {*** }}$ & 66.76 & 16.80 & 6.53 & $69.74^{* *}$ & 25.16 \\
\hline 76 & $\mathrm{GP}_{6}-442$ & $100.00^{* * *}$ & $92.50^{* * *}$ & $186.67^{\text {** }}$ & 70.14 & 20.39 & 9.93 & $83.66^{* * *}$ & 32.77 \\
\hline 77 & $\mathrm{GP}_{6}-451$ & $81.58^{* * *}$ & 39.47 & 46.96 & 20.93 & 13.60 & 8.34 & 29.90 & 14.49 \\
\hline 78 & $\mathrm{GP}_{6}-459$ & $85.00^{* * *}$ & 37.50 & 73.71 & 16.60 & 13.12 & 6.17 & 41.70 & 11.11 \\
\hline 79 & $\mathrm{GP}_{6}-511$ & 60.00 & 10.00 & 76.88 & 15.76 & 11.18 & 7.00 & 46.45 & 11.70 \\
\hline 80 & $\mathrm{GP}_{6}-517$ & 65.00 & 60.00 & 59.93 & 56.85 & 27.51 & 2.95 & 39.15 & 22.31 \\
\hline 81 & $\mathrm{GP}_{6}-534$ & 23.68 & 0.00 & 40.69 & 0.00 & 2.09 & 0.00 & 14.46 & 0.00 \\
\hline 82 & $\mathrm{GP}_{6}-561$ & $88.89^{* * *}$ & 58.33 & 62.80 & 29.09 & 22.38 & 8.56 & 45.44 & 20.28 \\
\hline 83 & $\mathrm{GP}_{6}-570$ & $75.00^{* * *}$ & 55.00 & 86.08 & 39.92 & 14.96 & 6.92 & 45.79 & 21.17 \\
\hline
\end{tabular}




\begin{tabular}{|c|c|c|c|c|c|c|c|c|c|}
\hline 84 & $\mathrm{GP}_{6}-578$ & $92.11^{* * *}$ & $76.32^{* * *}$ & $141.86^{* * *}$ & 59.55 & 13.95 & 5.10 & 49.15 & 20.08 \\
\hline 85 & $\mathrm{GP}_{6}-579$ & $90.00^{* * *}$ & 50.00 & 36.44 & 13.88 & 5.63 & 3.77 & 22.55 & 9.32 \\
\hline 86 & $\mathrm{GP}_{6}-586$ & 65.00 & 65.00 & $149.59^{* * *}$ & $109.83^{\text {*** }}$ & 26.56 & 17.05 & $85.22^{* * *}$ & $61.31^{* *}$ \\
\hline 87 & $\mathrm{GP}_{6}-589$ & 19.44 & 22.22 & $133.33^{* *}$ & $181.94^{\text {** }}$ & $31.47^{* *}$ & 12.18 & $58.64^{*}$ & $56.88^{*}$ \\
\hline 88 & $\mathrm{GP}_{6}-614$ & $97.50^{* * *}$ & $77.50^{* * *}$ & $104.31^{*}$ & 68.43 & 16.64 & 9.39 & 53.29 & 34.09 \\
\hline 89 & $\mathrm{GP}_{6}-615$ & 67.50 & 5.00 & 45.05 & 8.78 & 8.98 & 1.79 & 25.06 & 4.92 \\
\hline 90 & $\mathrm{GP}_{6}-656$ & $92.50^{* * *}$ & 22.50 & 78.88 & 31.18 & 11.65 & 8.29 & 45.35 & 19.77 \\
\hline 91 & $\mathrm{GP}_{6}-657$ & $77.50^{\text {*** }}$ & 0.00 & $148.68^{* * *}$ & 0.00 & 8.54 & 0.00 & $68.97^{* * *}$ & 0.00 \\
\hline 92 & $\mathrm{GP}_{6}-699$ & $77.50^{* *}$ & 67.50 & 69.07 & 19.40 & 11.21 & 5.56 & 44.73 & 13.58 \\
\hline 93 & $\mathrm{GP}_{6}-714$ & $85.00^{* * *}$ & $80.00^{* *}$ & $204.95^{* *}$ & $176.94^{* *}$ & $41.19^{* * *}$ & 9.65 & $107.39^{* *}$ & $77.31^{*: 3}$ \\
\hline 94 & $\mathrm{GP}_{6}-734$ & $92.50^{* * *}$ & 55.00 & $130.33^{* * *}$ & 72.48 & 16.06 & 5.24 & $69.27^{* *}$ & 36.53 \\
\hline 95 & $\mathrm{GP}_{6}-764$ & $72.22^{*}$ & 22.22 & 56.83 & 27.70 & 6.66 & 3.23 & 29.87 & 14.55 \\
\hline 96 & $\mathrm{GP}_{6}-792$ & $85.00^{* * *}$ & 37.50 & 85.49 & 21.23 & 14.50 & 5.54 & 52.15 & 13.93 \\
\hline 97 & $\mathrm{GP}_{6}-794$ & $75.00^{* * *}$ & 52.50 & 64.99 & 22.77 & 3.30 & 2.92 & 34.27 & 12.89 \\
\hline 98 & $\mathrm{GP}_{6}-819$ & 37.50 & 5.00 & 12.25 & 9.88 & 2.47 & 1.72 & 7.16 & 5.63 \\
\hline 99 & $\mathrm{GP}_{6}-847$ & 52.63 & 13.16 & 47.01 & 9.56 & 16.01 & 11.32 & 32.97 & 10.32 \\
\hline 100 & $\mathrm{GP}_{6}-854$ & $92.50^{* * *}$ & 67.50 & $108.11^{*}$ & 61.16 & 21.37 & 11.93 & $71.06^{* * *}$ & 40.14 \\
\hline 101 & $\mathrm{GP}_{6}-861$ & 33.33 & 0.00 & 26.66 & 0.00 & 2.65 & 0.00 & 16.41 & 0.00 \\
\hline 102 & $\mathrm{GP}_{6}-863$ & $92.50^{\text {*** }}$ & $92.50^{*}$ & 122.28 & 41.00 & 14.83 & 9.79 & $70.25^{* * i}$ & 25.89 \\
\hline 103 & $\mathrm{GP}_{6}-872$ & 58.82 & 0.00 & 62.12 & 0.00 & 17.91 & 0.00 & 39.07 & 0.00 \\
\hline 104 & $\mathrm{GP}_{6}-875$ & 40.00 & 7.50 & 62.99 & 9.02 & 4.88 & 3.25 & 27.92 & 5.60 \\
\hline 105 & $\mathrm{GP}_{6}-883$ & $72.50^{*}$ & 12.50 & 43.63 & 12.77 & 12.67 & 4.68 & 29.40 & 9.05 \\
\hline 106 & $\mathrm{GP}_{6}-887$ & 70.00 & 0.00 & $142.31^{*}$ & 0.00 & 17.46 & 0.00 & $81.43^{* * *}$ & 0.00 \\
\hline 107 & $\mathrm{GP}_{6}-891$ & 52.50 & 52.50 & 23.02 & 22.00 & 16.29 & 10.80 & 19.68 & 16.49 \\
\hline 108 & $\mathrm{GP}_{6}-899$ & 68.42 & 10.53 & 43.25 & 3.56 & 5.36 & 3.07 & 24.58 & 3.31 \\
\hline 109 & $\mathrm{GP}_{6}-906$ & 40.00 & 40.00 & 24.57 & 21.80 & 6.78 & 7.21 & 16.00 & 14.80 \\
\hline 110 & $\mathrm{GP}_{6}-912$ & $87.50^{* * *}$ & $72.50^{*}$ & $140.49^{* * *}$ & 68.15 & 18.77 & 5.59 & $78.41^{* *}$ & 36.24 \\
\hline 111 & $\mathrm{GP}_{6}-917$ & $88.89^{* * *}$ & 38.89 & 74.95 & 16.52 & 24.16 & 6.08 & 49.61 & 11.30 \\
\hline 112 & $\mathrm{GP}_{6}-951$ & 31.25 & 18.75 & 58.52 & 25.80 & 7.34 & 3.74 & 33.10 & 14.84 \\
\hline 113 & $\mathrm{GP}_{6}-952$ & 65.00 & 10.00 & 40.21 & 20.38 & 7.28 & 4.27 & 23.78 & 12.33 \\
\hline 114 & GP6-953 & 70.00 & 20.00 & 50.38 & 11.41 & 7.11 & 3.87 & 33.93 & 8.55 \\
\hline 115 & $\mathrm{GP}_{6}-961$ & 53.13 & 46.88 & 44.85 & 25.94 & 18.89 & 7.33 & 34.83 & 18.76 \\
\hline 116 & $\mathrm{GP}_{6}-965$ & $97.50^{\text {*** }}$ & 65.00 & 61.33 & 42.92 & 6.61 & 4.34 & 32.19 & 22.37 \\
\hline 117 & $\mathrm{GP}_{6}-967$ & $90.00^{* * *}$ & $80.00^{* * *}$ & $142.37^{* *}$ & 80.86 & 15.83 & 6.96 & $58.36^{*}$ & 31.86 \\
\hline 118 & $\mathrm{GP}_{6}-969$ & $90.00^{* * *}$ & $90.00^{* * *}$ & $120.76^{* * *}$ & 51.12 & 20.95 & 7.05 & 55.43 & 22.33 \\
\hline 119 & $\mathrm{GP}_{6}-990$ & 45.00 & 15.00 & 32.53 & 19.28 & 8.45 & 5.43 & 17.73 & 10.76 \\
\hline 120 & $\mathrm{GP}_{6}-1001$ & $81.58^{\text {*** }}$ & 47.37 & 38.60 & 16.86 & 8.64 & 6.34 & 25.63 & 12.30 \\
\hline 121 & $\mathrm{GP}_{6}-1020$ & $82.50^{* * *}$ & 17.50 & 45.60 & 33.04 & 6.86 & 5.06 & 28.80 & 20.90 \\
\hline 122 & $\mathrm{GP}_{6}-1023$ & $89.47^{* * *}$ & 57.89 & $130.53^{* * *}$ & 30.20 & 14.14 & 4.20 & $58.21^{*}$ & 14.05 \\
\hline 123 & $\mathrm{GP}_{6}-1026$ & $75.00^{* * *}$ & 0.00 & 32.66 & 0.00 & 7.62 & 0.00 & 17.50 & 0.00 \\
\hline 124 & $\mathrm{GP}_{6}-1037$ & $81.58^{\text {*** }}$ & 34.21 & $154.44^{* * *}$ & 48.16 & 9.44 & 4.08 & $62.97^{* * *}$ & 20.34 \\
\hline 125 & $\mathrm{GP}_{6}-1047$ & $90.00^{* * *}$ & 52.50 & 87.96 & 11.42 & 11.19 & 4.79 & 37.06 & 7.05 \\
\hline 126 & $\mathrm{GP}_{6}-1060$ & $95.00^{* * *}$ & $80.00^{* * *}$ & $166.48^{* * *}$ & 74.60 & 13.47 & 9.44 & $69.68^{* *}$ & 33.30 \\
\hline 127 & $\mathrm{GP}_{6}-1063$ & $90.00^{* * *}$ & 20.00 & 62.68 & 26.97 & 5.03 & 3.41 & 26.43 & 12.15 \\
\hline 128 & $\mathrm{GP}_{6}-1072$ & $80.00^{* *}$ & $75.00^{* *}$ & 60.95 & 61.78 & 7.59 & 4.54 & 31.83 & 30.57 \\
\hline
\end{tabular}




\begin{tabular}{|c|c|c|c|c|c|c|c|c|c|}
\hline 129 & $\mathrm{GP}_{6}-1075$ & $90.00^{* * *}$ & 57.50 & $154.14^{* * *}$ & 77.26 & 17.36 & 5.56 & $65.27^{* *}$ & 30.68 \\
\hline 130 & $\mathrm{GP}_{6}-1089$ & 12.50 & 0.00 & 7.12 & 0.00 & 7.47 & 0.00 & 7.28 & 0.00 \\
\hline 131 & $\mathrm{GP}_{6}-1101$ & $82.50^{* * *}$ & 40.00 & $142.31^{\text {*** }}$ & 56.87 & 22.76 & 13.20 & $84.35^{* * *}$ & 35.57 \\
\hline 132 & $\mathrm{GP}_{6}-1102$ & $82.50^{* * *}$ & $75.00^{* * *}$ & $153.13^{* *}$ & 92.62 & $33.59^{\text {*** }}$ & 13.70 & $90.35^{* * *}$ & 51.18 \\
\hline 133 & $\mathrm{GP}_{6}-1114$ & $76.84^{* * *}$ & 18.03 & 66.59 & 9.60 & 18.28 & 9.62 & 51.65 & 9.58 \\
\hline 134 & $\mathrm{GP}_{6}-1117$ & $87.50^{* * *}$ & 35.00 & $153.47^{* *}$ & 32.15 & $30.72^{* * *}$ & 8.97 & $87.29^{* * *}$ & 19.65 \\
\hline 135 & $\mathrm{GP}_{6}-1127$ & 57.50 & 7.50 & 61.24 & 6.17 & 8.79 & 9.34 & 35.71 & 7.71 \\
\hline 136 & $\mathrm{GP}_{6}-1135$ & 57.50 & 0.00 & 23.71 & 0.00 & 6.28 & 0.00 & 16.61 & 0.00 \\
\hline 137 & $\mathrm{GP}_{6}-1150$ & 55.56 & 19.44 & 54.37 & 24.77 & 8.13 & 6.94 & 29.70 & 15.26 \\
\hline 138 & $\mathrm{GP}_{6}-1207$ & $90.00^{* *}$ & 20.00 & 52.31 & 8.18 & 8.96 & 3.25 & 32.86 & 5.97 \\
\hline 139 & $\mathrm{GP}_{6}-1217$ & $92.11^{* * *}$ & 10.53 & 39.18 & 1.53 & 9.76 & 1.88 & 23.32 & 1.72 \\
\hline 140 & $\mathrm{GP}_{6}-1227$ & 36.11 & 13.89 & 52.20 & 2.29 & 15.92 & 7.10 & 37.97 & 4.18 \\
\hline 141 & $\mathrm{GP}_{6}-1228$ & $78.95^{* * *}$ & $71.05^{*}$ & 59.34 & 22.62 & 18.41 & 3.70 & 43.57 & 15.33 \\
\hline 142 & $\mathrm{GP}_{6}-1254$ & $88.89^{* * *}$ & 69.44 & 95.93 & 60.09 & 13.77 & 7.29 & 55.44 & 34.07 \\
\hline 143 & $\mathrm{GP}_{6} 1350$ & 32.08 & 16.25 & 35.31 & 32.55 & 17.87 & 9.95 & 26.14 & 20.70 \\
\hline 144 & $\mathrm{GP}_{6} 1436$ & 61.46 & 32.50 & 69.39 & 13.87 & 10.02 & 5.24 & 43.94 & 10.18 \\
\hline 145 & $\mathrm{GP}_{6} 1450$ & 15.00 & 0.00 & 10.01 & 0.00 & 3.58 & 0.00 & 6.12 & 0.00 \\
\hline 146 & $\mathrm{GP}_{6}-1468$ & $87.50^{\text {*** }}$ & 32.50 & $247.51^{* * *}$ & 77.62 & 17.48 & 9.21 & $112.88^{* * *}$ & 37.63 \\
\hline 147 & $\mathrm{GP}_{6}-1477$ & $80.00^{\text {*** }}$ & 32.50 & $106.95^{*}$ & 66.86 & 19.38 & 9.85 & 52.70 & 31.52 \\
\hline 148 & $\mathrm{GP}_{6} 1482$ & $80.56^{* * *}$ & 33.33 & $119.51^{* * *}$ & 64.71 & $29.58^{*}$ & 20.49 & $63.94^{* *}$ & 37.34 \\
\hline 149 & $\mathrm{GP}_{6}-1509$ & 69.44 & 38.89 & 86.56 & 30.55 & 15.62 & 6.27 & 51.13 & 18.45 \\
\hline 150 & $\mathrm{GP}_{6}-1518$ & $87.50^{* * *}$ & 35.00 & 53.93 & 8.53 & 10.75 & 5.61 & 36.28 & 7.34 \\
\hline 151 & $\mathrm{GP}_{6}-1533$ & $83.33^{* * *}$ & 63.89 & $120.34^{* * *}$ & 58.30 & 14.38 & 7.94 & $63.86^{* * *}$ & 31.47 \\
\hline 152 & $\mathrm{GP}_{6}-1561$ & $82.50^{* * *}$ & 35.00 & 76.12 & 19.27 & 14.58 & 5.55 & 46.05 & 12.57 \\
\hline 153 & $\mathrm{GP}_{6}-1573$ & $95.00^{* * *}$ & 45.00 & 95.79 & 54.84 & 11.68 & 7.11 & 54.60 & 31.48 \\
\hline 154 & $\mathrm{GP}_{6}-1576$ & $95.00^{* * *}$ & $85.00^{* * *}$ & $120.45^{* *}$ & 50.22 & 22.48 & 12.50 & $72.75^{\text {** }}$ & 31.98 \\
\hline 155 & $\mathrm{GP}_{6}-1588$ & 55.26 & 7.89 & 54.18 & 18.96 & 8.74 & 7.90 & 27.73 & 12.52 \\
\hline 156 & $\mathrm{GP}_{6}-1595$ & $75.00^{* * *}$ & 65.00 & 51.48 & 40.75 & 9.83 & 8.59 & 27.10 & 21.90 \\
\hline 157 & $\mathrm{GP}_{6}-1616$ & $95.00^{* * *}$ & 70.00 & 84.91 & 66.48 & 19.90 & 5.31 & 49.02 & 32.71 \\
\hline 158 & $\mathrm{GP}_{6}-1665$ & $95.00^{* * *}$ & 27.50 & 34.95 & 6.83 & 8.75 & 8.58 & 22.82 & 7.63 \\
\hline 159 & $\mathrm{GP}_{6}-1725$ & $85.00^{* * *}$ & 60.00 & 97.09 & 60.59 & 22.29 & 15.26 & $65.88^{* * *}$ & 41.60 \\
\hline 160 & $\mathrm{GP}_{6}-2255$ & $100.00^{* * *}$ & $95.00^{* * *}$ & 91.09 & 42.44 & 20.84 & 12.01 & $59.75^{* *}$ & 28.86 \\
\hline & Range & \multicolumn{2}{|c|}{$0.00-100.00$} & \multicolumn{2}{|c|}{$0.00-744.41$} & \multicolumn{2}{|c|}{ 0.00-93.72 } & \multicolumn{2}{|c|}{$0.00-282.85$} \\
\hline \multicolumn{2}{|c|}{ Overall Mean } & \multicolumn{2}{|c|}{61.86} & \multicolumn{2}{|c|}{82.64} & \multicolumn{2}{|c|}{21.34} & \multicolumn{2}{|c|}{48.86} \\
\hline \multicolumn{2}{|r|}{ S.Em+/- } & \multicolumn{2}{|c|}{3.08} & \multicolumn{2}{|c|}{7.13} & \multicolumn{2}{|c|}{2.30} & \multicolumn{2}{|c|}{2.73} \\
\hline \multicolumn{2}{|r|}{ CD 5\% } & \multicolumn{2}{|c|}{8.54} & \multicolumn{2}{|c|}{19.76} & \multicolumn{2}{|c|}{6.38} & \multicolumn{2}{|c|}{7.57} \\
\hline \multicolumn{2}{|r|}{ CD $1 \%$} & \multicolumn{2}{|c|}{11.23} & \multicolumn{2}{|c|}{26.00} & \multicolumn{2}{|c|}{8.40} & \multicolumn{2}{|c|}{9.96} \\
\hline & $\mathrm{CV}$ & \multicolumn{2}{|c|}{7.03} & & & & & & \\
\hline
\end{tabular}

* - significant at $5 \%$ probability; $* *$ - significant at $1 \%$ probability

The investigation strongly agreed to use PEG6000 for inducing as well as selection of genotypes for moisture stress tolerance under in vitro condition. Based on overall mean performance of stress tolerance indices like,
GTI, RLTI, PHTI and SLTI, there are 34 promising genotypes were identified viz., CMS 857B, CMS 104B, CMS 148B, R 78, R12-2, GP $\mathrm{GP}_{6}-11, \mathrm{GP}_{6}-54, \mathrm{GP}_{6}-118, \mathrm{GP}_{6}-211$, $\mathrm{GP}_{6}-305, \mathrm{GP}_{6}-310, \mathrm{GP}_{6}-325, \mathrm{GP}_{6}-326, \mathrm{GP}_{6^{-}}$ 
366, $\mathrm{GP}_{6}-370, \mathrm{GP}_{6}-371, \mathrm{GP}_{6}-424, \mathrm{GP}_{6}-442$, $\mathrm{GP}_{6}-578, \mathrm{GP}_{6}-586, \mathrm{GP}_{6}-589, \mathrm{GP}_{6}-614, \mathrm{GP}_{6-}$ 714, GP 6 -734, GP $6-863, \mathrm{GP}_{6}-912, \mathrm{GP}_{6}-967$, $\mathrm{GP}_{6}-969, \mathrm{GP}_{6}-1060, \mathrm{GP}_{6}-1072, \mathrm{GP}_{6}-1102$, $\mathrm{GP}_{6}-1228, \mathrm{GP}_{6}-1576$ and $\mathrm{GP}_{6}-2255$. These were found to be most tolerant lines can be utilized for further breeding programs to improve the genotypes to overcome drought stress at germination stage.

Acknowledgement: This research was supported/partially supported by Associate Director of Research. We thank, my advisory committee members from UAS, Raichur, who provided insight and expertise that greatly assisted in the research.

\section{References}

Ashraf, M. and Mehmood, S. 1990. Response of four Brassica species to drought stress. Environ. Expt. Bot., 30: 93-100.

Ashraf, M.Y., Akhtar, K. Hussain F.and Iqbal. J. 2006. Screening of different accessions of three potential grass species from Cholistan desert for salt tolerance. Pak. J. Bot., 38: 1589-1597.

Ahmad, S., Ahmad, R., Ashraf, M.Y., Ashraf,
M. and Waraich, E.A. 2009. Sunflower (Helianthus annuusL.) response to drought stress at germination and seedling growth stages. Pak. J. Bot., 41(2): 647-654.

Geetha, A., Sivasankar, A., Lakshmi Prayaga, Suresh, J. and Saidaiah, P. 2012. Screening of sunflower genotypes for drought tolerance under laboratory conditions using PEG. Sabrao J. Breed. Genet., 44(1): 28-41.

Saensee, K., Machikowa, T. and Muangsan, N. 2011. Evaluation of drought response of sunflower synthetic varieties / lines. International symposium on sunflower genetic resources, October 16 - 20, Kuşadasi, Izmir, Turkey.

Saensee, K., Machikowa, T. and Muangsan, N. 2012. Comparative performance of Sunflower synthetic varieties under drought stress. Int. J. Agric. Biol., 4(6): 929-934.

Turhan, H. and Baser, I. 2004. In-vitro and Invivo water stress in Sunflower (Helianthus annuus L.). Helia, 27(40): 227-236.

\section{How to cite this article:}

Sheshaiah, I. Shankergoud, R. Siddhesh, Nadkarni and Vikas V. Kulakarni. 2017. Screening of Sunflower (Helianthus annuus L) Genotypes for Moisture Stress Tolerance using PEG-6000. Int.J.Curr.Microbiol.App.Sci. 6(5): 848-856. doi: https://doi.org/10.20546/ijcmas.2017.605.095 